\title{
BMJ Open Interventions to improve screening and appropriate referral of patients with cancer for psychosocial distress: systematic review
}

\author{
Kristen McCarter, ${ }^{1}$ Ben Britton, ${ }^{2}$ Amanda L Baker, ${ }^{2}$ Sean A Halpin, ${ }^{1}$ Alison K Beck, ${ }^{2}$ \\ Gregory Carter, ${ }^{2}$ Chris Wratten, ${ }^{3}$ Judith Bauer, ${ }^{4}$ Erin Forbes, ${ }^{2}$ Debbie Booth, ${ }^{5}$ \\ Luke Wolfenden ${ }^{2}$
}

To cite: McCarter K,

Britton B, Baker AL, et al. Interventions to improve screening and appropriate referral of patients with cancer for psychosocial distress: systematic review. BMJ Open 2018;8:e017959. doi:10.1136/ bmjopen-2017-017959

- Prepublication history and additional material for this paper are available online. To view these files, please visit the journal online (http://dx.doi. org/10.1136/bmjopen-2017017959).

Received 27 May 2017 Revised 6 November 2017 Accepted 16 November 2017

\section{CrossMark}

${ }^{1}$ School of Psychology, University of Newcastle, Callaghan,

Australia

${ }^{2}$ School of Medicine and Public Health, University of Newcastle, Callaghan, Australia

${ }^{3}$ Department of Radiation Oncology, Calvary Mater

Newcastle Hospital, Waratah,

Australia

${ }^{4}$ Centre for Dietetics Research, University of Queensland, St Lucia, Australia

${ }^{5}$ University Library, University of Newcastle, Callaghan, Australia

Correspondence to Kristen McCarter; Kristen.McCarter@newcastle. edu.au

\section{ABSTRACT}

Objectives The primary aim of the review was to determine the effectiveness of strategies to improve clinician provision of psychosocial distress screening and referral of patients with cancer.

Design Systematic review.

Data sources Electronic databases (Cochrane Central Register of Controlled Trials, MEDLINE, EMBASE, PsycINF0 and Cumulative Index to Nursing and Allied Health Literature (CINAHL)) were searched until July 2016. Inclusion criteria Population: adult patients with cancer and clinical staff members. Intervention: any strategy that aimed to improve the rate of routine screening and referra for detected distress of patients with cancer. Comparison: no intervention controls, 'usual' practice or alternative interventions. Outcome: (primary) any measure of provision of screening and/or referral for distress, (secondary) psychosocial distress, unintended adverse effects. Design: trials with or without a temporal comparison group, including randomised and non-randomised trials, and uncontrolled pre-post studies.

Data extraction and analysis Two review authors independently extracted data. Heterogeneity across studies precluded quantitative assessment via meta-analysis and so a narrative synthesis of the results is presented. Results Five studies met the inclusion criteria. All studies were set in oncology clinics or departments and used multiple implementation strategies. Using the Grades of Recommendation, Assessment, Development and Evaluation, the overall rating of the certainty of the body of evidence reported in this review was assessed as very low. Three studies received a methodological quality rating of weak and two studies received a rating of moderate. Only one of the five studies reported a significant improvement in referrals.

Conclusions The review identified five studies of predominantly poor quality examining the effectiveness of strategies to improve the routine implementation of distress screening and referral for patients with cancer. Future research using robust research designs, including randomised assignment, are needed to identify effective support strategies to maximise the potential for successful implementation of distress screening and referral for patients with cancer.

PROSPERO registration number CRD42015017518.
Strengths and limitations of this study

- This is the first review to systematically synthesise evidence of the effectiveness of strategies to improve the rate of routine distress screening and referral for patients with cancer.

- The review performed a comprehensive search of the literature, included controlled trials of any design and was inclusive of non-English literature.

- Few studies met the inclusion criteria, and heterogeneity of study design, primary and secondary outcomes precluded quantitative synthesis.

\section{INTRODUCTION}

\section{Rationale}

Psychosocial distress can be defined as an unpleasant experience of an emotional or psychological nature, including depression, anxiety and other $/ \operatorname{mood} /$ adjustment disorders. ${ }^{1}$ Estimates of the prevalence of psychosocial distress vary due to the type and stage of cancer, patient age, gender and race, as well as the definition of distress used. Psychosocial distress can arise in response to cancer-related factors such as diagnosis and cancer progression, pain and adverse effects of treatment. Psychosocial distress in patients with cancer may lead to non-adherence to treatment, poorer quality of life and may negatively impact survival, as well as increase treatment burden to the oncology team and health system. ${ }^{1-4}$ Therefore, recognising and treating distress in cancer populations are an important health priority.

Professional associations and clinical guidelines including the National Comprehensive Cancer Network (NCCN) Clinical Practice Guidelines in Oncology: Distress Management ${ }^{1}$ recommend that those responsible for the 
care of patients with cancer routinely screen for distress and, as appropriate, refer for further assessment and support. Clinical practice guideline recommendations are based on evidence that screening improves the timely management of distress, ${ }^{3}{ }^{5}$ and on systematic reviews and meta-analyses that have demonstrated psychosocial intervention reduces distress (such as depression and anxiety ${ }^{6}$ ), particularly when participants are prescreened. $^{8}$

The efficacy of distress screening for improving patient outcomes has been challenged in the literature. A recent systematic review failed to find evidence that distress screening improved distress outcomes among patients with cancer. ${ }^{9}$ Another systematic review that examined screening for distress in cancer settings found that those studies reporting a lack of benefit to distress screening in patients with cancer lacked appropriate follow-up care of distressed patients, while trials that linked screening with mandatory referral or intervention showed improvement in patient outcomes. ${ }^{10}$ While screening itself may not be sufficient to improve patient outcomes, it is a necessary prerequisite to identify those patients who could benefit from evidencebased treatment and guides clinical decision making. ${ }^{1}$ Consequently, clinical guidelines recommend screening and referral protocols in cancer settings. It is clear that well-designed trials are needed to further evaluate the effectiveness of screening and referral on patient outcomes. However, in the absence of strong evidence from robust trials that suggests distress screening and referral should not be conducted, clinicians should be guided by clinical practice guidelines.

Despite clinical practice guideline recommendations, screening and referral of patients with cancer for psychosocial distress are not routinely conducted by clinicians responsible for the clinical management of cancer. ${ }^{1211}$ Beginning in 2015, the American College of Surgeons Commission on Cancer has required cancer centres to implement programmes for distress screening as a criterion for accreditation. ${ }^{12}$ A recent cross-sectional survey of 20 NCGN institutions reported only $60 \%$ of services conducted outpatient distress screening, and even fewer services reported screening all patients $(30 \%)$ as outlined in the NCCN standards. ${ }^{11}$ Systematic reviews of trials of strategies to improve depression or anxiety screening in primary care note that complex organisational interventions that incorporate multiple strategies are most effective in improving provision of care. ${ }^{13-15}$ Such strategies include clinician education, opinion leaders, patient-specific reminders, enhanced role of nurses, academic detailing, integrating screening into routine clinical reviews and a greater degree of coordination between services (eg, between primary and secondary care). ${ }^{13-15}$ However, we are not aware of any previous systematic review of interventions to improve clinician routine provision of distress screening and appropriate referral of patients with cancer per se. It is the discrepancy between these guideline recommendations and current practice that this review aims to address.

\section{Objectives}

The primary aim of this review was to assess for patients with cancer the impact of trials of strategies to improve clinician delivery of psychosocial distress care compared with usual care on rates of psychosocial distress screening and referral for further assessment and/or psychosocial support.

The following were the secondary aims of the review:

i. To describe the effectiveness of such interventions on reducing psychosocial distress of patients with cancer.

ii. To describe any unintended adverse effects of such an intervention.

\section{METHODS AND ANALYSIS}

The review will be reported consistent with the Preferred Reporting Items for Systematic Reviews and Meta-Analyses statement. ${ }^{16}$ The details of the methods have been reported elsewhere ${ }^{17}$ and the protocol is registered with PROSPERO (registration number CRD42015017518).

\section{Eligibility criteria}

Study characteristics

Types of studies

Original studies including randomised controlled trials and non-randomised trials were included. Exclusion criteria were trials without parallel comparison or control groups. Due to the limited number of studies (explained further in the Results section), we later included studies without parallel control groups including uncontrolled pre-post studies. There were no restrictions based on length of follow-up, year of study publication or language. Studies could be published in peer review or grey literature.

\section{Participants}

Participants could include adult patients with cancer and clinical staff members such as physicians and allied health professionals responsible for the care of patients with cancer. Studies that examined screening for psychosocial distress and/or referral for carers of patients with cancer, or survivors of cancer, were excluded.

\section{Types of interventions}

Interventions of strategies that aimed to improve the rate of screening procedures for psychosocial distress and/or rate of referral for appropriate psychosocial support in healthcare settings were included. There are a range of potential strategies that could improve the likelihood of implementation of distress screening and referral in healthcare settings. For example, the Cochrane Effective Practice and Organisation of Care (EPOC) taxonomy is a framework for characterising 
educational, behavioural, financial, regulatory and organisational interventions within the topic of 'implementation strategies" ${ }^{18}$ and includes 22 subcategories. Examples of strategies within the taxonomy include educational materials, performance monitoring, local consensus processes and educational outreach visits. Included interventions could be singular or multicomponent. Studies using clinical judgement of psychosocial distress alone, without use of a formal screening tool, were excluded. Referral for psychosocial support was defined as any written or verbal offer or direction of a patient for further review, consultation, assessment or treatment with any health professional, including the primary oncology team or health service, offering psychosocial support such as psycho-oncology services. Studies were included if they implemented either distress screening only or distress screening and appropriate referral. Studies where research staff conduct screening or referral were excluded.

\section{Comparisons}

Studies with no intervention controls, 'usual' practice periods or alternative intervention comparison groups were included.

\section{Outcomes}

\section{Primary outcomes}

1. Any measure of the provision of screening for psychosocial distress (eg, number or $\%$ of patients with cancer screened).

2. Any measure of the provision of referral for further assessment and/or psychosocial support (eg, number or $\%$ of patients with cancer referred) by a clinician responsible for the management of a patient with cancer.

\section{Secondary outcomes}

1. Any validated outcome measure of change in psychosocial distress levels in patients (eg, distress outcome assessments such as the Kessler Psychological Distress Scale).

2. Any measure of adverse effects on patients, clinicians or health services; or barriers to performing screening such as displacement of other clinical priorities.

\section{Information sources}

\section{Electronic databases}

The following electronic databases were searched for potentially eligible studies published up until July 2016: the Cochrane Central Register of Controlled Trials in the Cochrane Library, MEDLINE, EMBASE, PsycINFO and Cumulative Index to Nursing and Allied Health Literature (CINAHL). The MEDLINE search strategy (online supplementary file) was adapted for other databases and included filters used in other systematic reviews for population (cancer patients), ${ }^{19}$ screening for distress ${ }^{20}$ and referral $^{21}$ and psychosocial support. ${ }^{22}$

\section{Other sources}

Studies were also obtained from the following sources:

- Reference lists of included studies.

- Hand-searching of three relevant journals in the field (published in the last 5 years): Journal of the National Comprehensive Cancer Network, Psycho-Oncology and Supportive Care in Cancer.

- Hand-searching of conference abstracts published in the preceding 2 years from the International Psycho-Oncology Society and the Society of Behavioral Medicine.

- A grey literature search using Google Scholar (published online in the last 5 years-the first 200 citations were examined).

\section{Study selection}

The titles and abstracts retrieved by electronic searches were exported to a reference management database (EndNote V.X6) to remove duplicates. Two reviewers independently screened abstracts and titles using a standardised screening tool that was pilot-tested with a sample of articles before use. The abstracts of papers that were in a language other than English were translated using Google Translate. If considered eligible or eligibility was unclear, professional translation of the full paper was undertaken.

The full texts of manuscripts were obtained for all potentially eligible trials for further examination and independently screened by two reviewers. For all manuscripts, the primary reason for exclusion was recorded and is documented in figure 1. Discrepancies regarding study eligibility were resolved by discussion and consensus.

\section{Data extraction}

Two review authors (KM and EF) independently extracted data from the included trials using a prepiloted data extraction form that was developed based on recommendations from the Cochrane Handbook for Systematic Reviews of Interventions. ${ }^{23}$ Discrepancies regarding data extraction were resolved by discussion and consensus.

\section{Data items}

Data were sought for the following variables:

- Authors, year and journal.

- Study eligibility, study design, healthcare provider type (eg, nurses), country and healthcare setting (eg, oncology clinic).

- Patient characteristics and demographics, including cancer site, cancer stage, age, sex, cancer treatment type and treatment status (pre/undergoing/post).

- Characteristics of the intervention, including the duration, intervention strategies and screening instrument.

- Trial primary and secondary outcomes, including sample size, the data collection method, validity of measures used, any measures of client uptake or use 


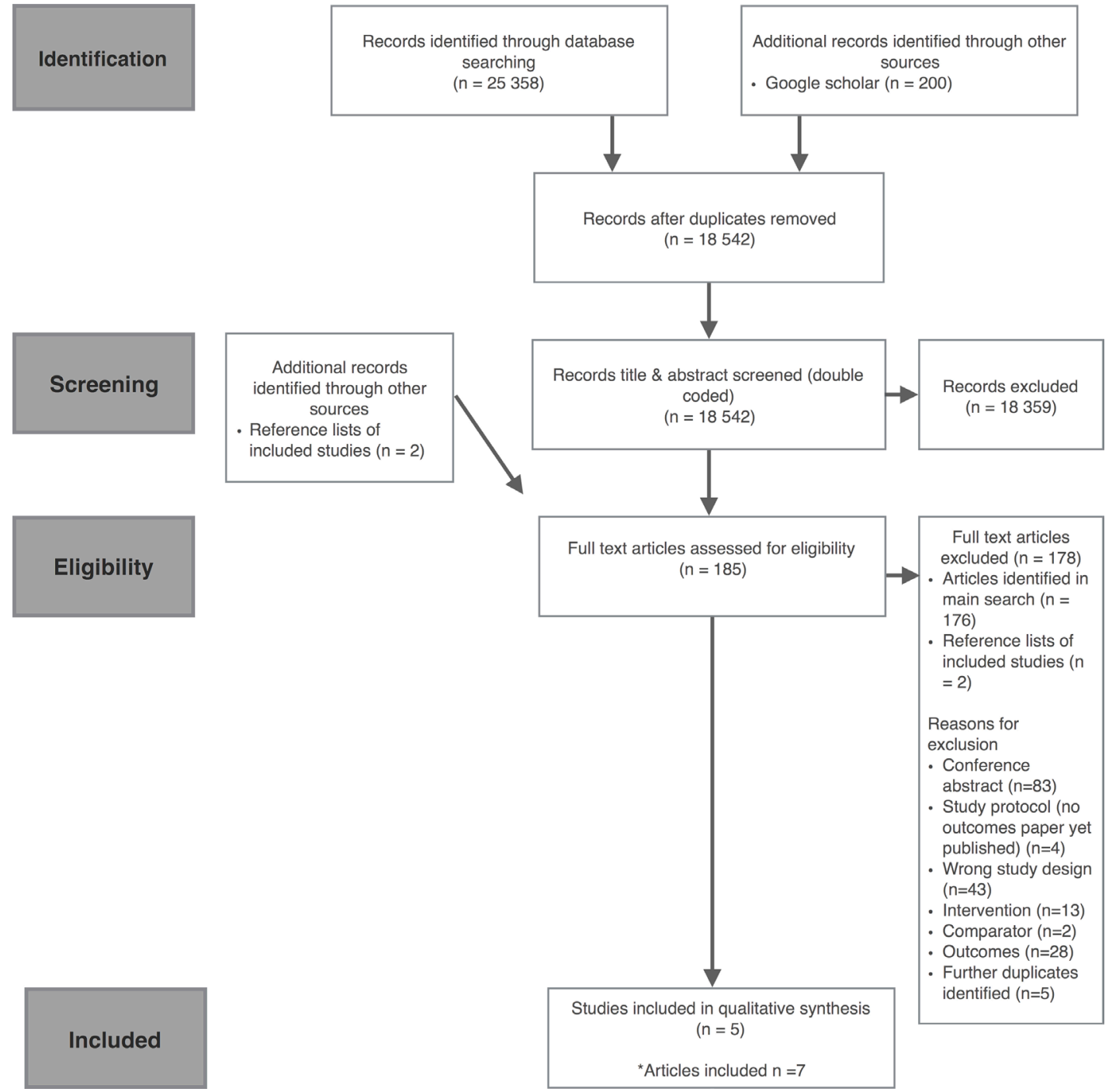

Figure 1 Preferred Reporting Items for Systematic Reviews and Meta-Analyses flow diagram.

of psychosocial support services following referral, effect size, and measures of change in distress.

- Number of participants per experimental condition.

- Information to allow assessment of risk of study bias.

\section{Methodological quality assessment bias}

Two review authors (KM and EF) independently assessed the risk of bias of all included trials using the Effective Public Health Practice Project (EPHPP) Quality Assessment Tool for quantitative studies. ${ }^{24}$ The use of the EPHPP tool was a post-hoc change from protocol due to the study designs included in the review. This tool covers any quantitative study design and includes components of intervention integrity. Any discrepancies were resolved through discussion. The EPHPP assesses six methodological dimensions: selection bias, study design, confounders, blinding, data collection methods, and withdrawals and dropouts. These domains are rated on a 3-point scale (strong, moderate, weak) according to predefined criteria and procedures recommended for tool use, and then given an overall global rating. Those with no weak ratings were given an overall rating of strong, those with one weak rating were given an overall rating of moderate and those with two or more weak ratings across the six domains were given an overall weak rating. Two additional methodological dimensions provided by the tool are intervention integrity and analyses, and these were also completed by the reviewers.

\section{Data analysis}

Summary measures

The small number of studies and differences in study design and primary and secondary outcomes reported in the included studies precluded the use of summary statistics to describe treatment effects. As such, the findings of included trials are described narratively.

\section{Grading the strength of evidence}

As recommended by the Cochrane Handbook for Systematic Reviews of Interventions, ${ }^{23}$ the overall quality of evidence on primary outcomes is presented using the GRADE (Grades of Recommendation, Assessment, Development and Evaluation) approach, which involves consideration 
of within-study risk of bias (methodological quality), directness of evidence, heterogeneity, precision of effect estimates and risk of publication bias. The overall quality of evidence was rated by two review authors (KM and EF) at four levels: high, moderate, low and very low.

\section{RESULTS}

A total of 18542 citations were identified (after duplicates were removed) (figure 1) for abstract and title screening. Just one study met the eligibility criteria (ie, parallel control/comparison group). As such, and in an attempt to provide some evidence to guide researchers and practitioners regarding methods to improve patient distress screening and referral of patients with cancer, we relaxed the design criteria and post-hoc rescreened all 18542 citations and included studies with controlled trial designs without parallel control groups including uncontrolled pre-post studies. The full text of 185 manuscripts were sought for further assessment against the review inclusion criteria (figure 1). Of these, 178 were considered ineligible following the trial screening process. Seven publications describing five trials were included in the review.

\section{Included studies}

Types of studies

A description of the trial characteristics of included studies is provided in table 1 . One study was conducted in Japan, ${ }^{25}$ one in the Netherlands, ${ }^{26-28}$ one in Germany, ${ }^{29}$ one in Belgium ${ }^{30}$ and one in Australia. ${ }^{31}$ Studies were published between 2009 and 2014. There was considerable heterogeneity in the participants, interventions and outcomes (clinical heterogeneity) of included studies.

\section{Health providers}

All studies were set in oncology clinics or departments. In regard to the healthcare providers responsible for conducting the distress screening and/or referral, one study targeted nurses, ${ }^{31}$ one targeted radiation oncologists, ${ }^{26-28}$ one required pharmacists to perform the screening, ${ }^{25}$ one study involved both specialised breast care nurses and doctors ${ }^{29}$ and one study used oncologists. ${ }^{30}$

\section{Interventions}

All trials used multiple implementation strategies. The EPOC subcategories used to classify the implementation strategies employed by included studies in the review are provided in table 2. The interventions employed in the included studies, as well as the specific EPOC subcategories identified in each study, are presented in table 3. Using EPOC taxonomy descriptors, all trials included educational materials and educational meetings, with two trials using only these strategies. ${ }^{30} 31$ One trial used these strategies with the addition of educational outreach visits. ${ }^{26-28}$ One study used a combination of educational materials, educational meetings, educational outreach visits and reminders. ${ }^{25}$ One study tested an intervention consisting of organisational culture, continuous quality improvement, educational materials, educational meetings and reminders. ${ }^{29}$

\section{Outcomes}

The primary and secondary outcomes are presented in tables 4 and 5. Implementation of distress screening and/or referral was primarily assessed using reviews of patient medical records ${ }^{25-29} 31$; however, one study did not report the data collection method. ${ }^{28}$ None of the studies reported which staff completed the medical record reviews. All trials reported the rates of referral for support for those patients identified as distressed; however, none of the studies examined the improvement in rates of distress screening. Change in distress levels was reported in one study. ${ }^{26-28}$ No study included a measure of potential adverse effects.

\section{Study design characteristics}

One of the included studies was a cluster randomised controlled trial, ${ }^{26-28}$ three were pre-post studies ${ }^{25} 3031$ and one was a prospective consecutive study. ${ }^{29}$ The cluster randomised controlled trial compared an intervention with a usual care control, ${ }^{26-28}$ three studies compared a screening programme period with a usual care period, ${ }^{253031}$ and one trial compared a screening programme phase with a two-phase non-screening period. ${ }^{29}$

\section{Methodological quality assessment}

Individual ratings for each study against the six methodological criteria from the EPHPP tool and the assigned global rating are reported in table 6 . Overall, three studies received a methodological quality rating of weak ${ }^{29-31}$ and two studies received a rating of moderate. ${ }^{25-28}$ For three of the four non-randomised studies, ${ }^{29-31}$ it was unclear whether confounders were adequately adjusted for, and for the majority of studies blinding of outcome assessors or study participants was not described. While most studies reported medical record reviews for the data collection method, no reference was made to their validity or reliability as an outcome measure, nor was a description of who conducted the audits provided, resulting in weak ratings for all studies. All studies were judged as using analyses as appropriate to study design.

\section{Effects of intervention on distress screening and/or referral}

None of the included trials reported on the effects of strategies to improve rates of distress screening provision. Only one of the five studies reported a significant improvement in rate of referrals. ${ }^{27}$ Zemlin et $a l^{29}$ reported a significant positive trend for the proportion of patients who were informed/offered psycho-oncological interview $(\mathrm{t}=22.40, \mathrm{df}=2, \mathrm{P}<0.001)$. The effects of interventions are presented according to the implementation strategies (classified using the EPOC taxonomy) employed by included studies.

\section{Educational materials and educational meetings}

Two studies examined the impact of educational materials and educational meetings only on distress 


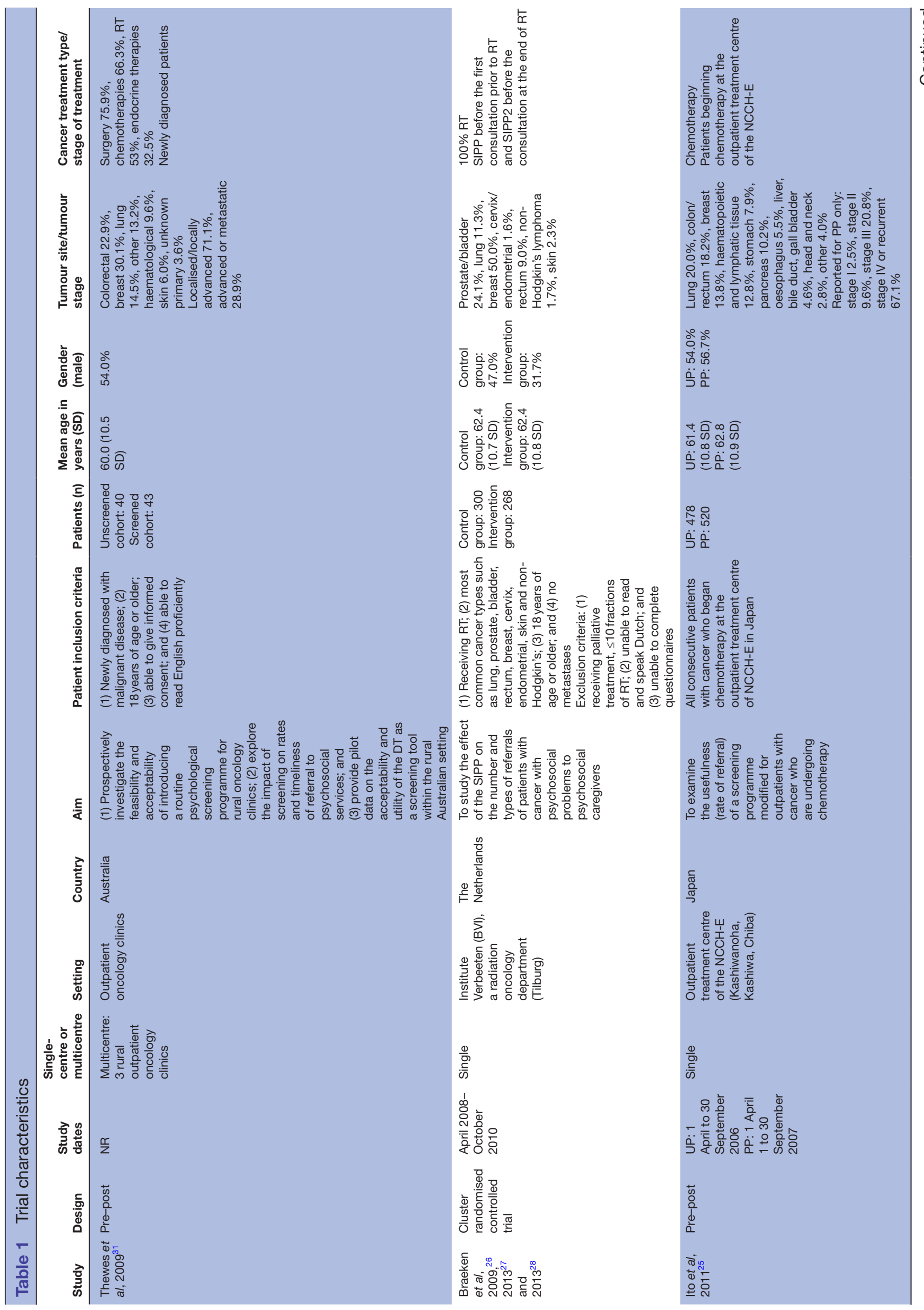




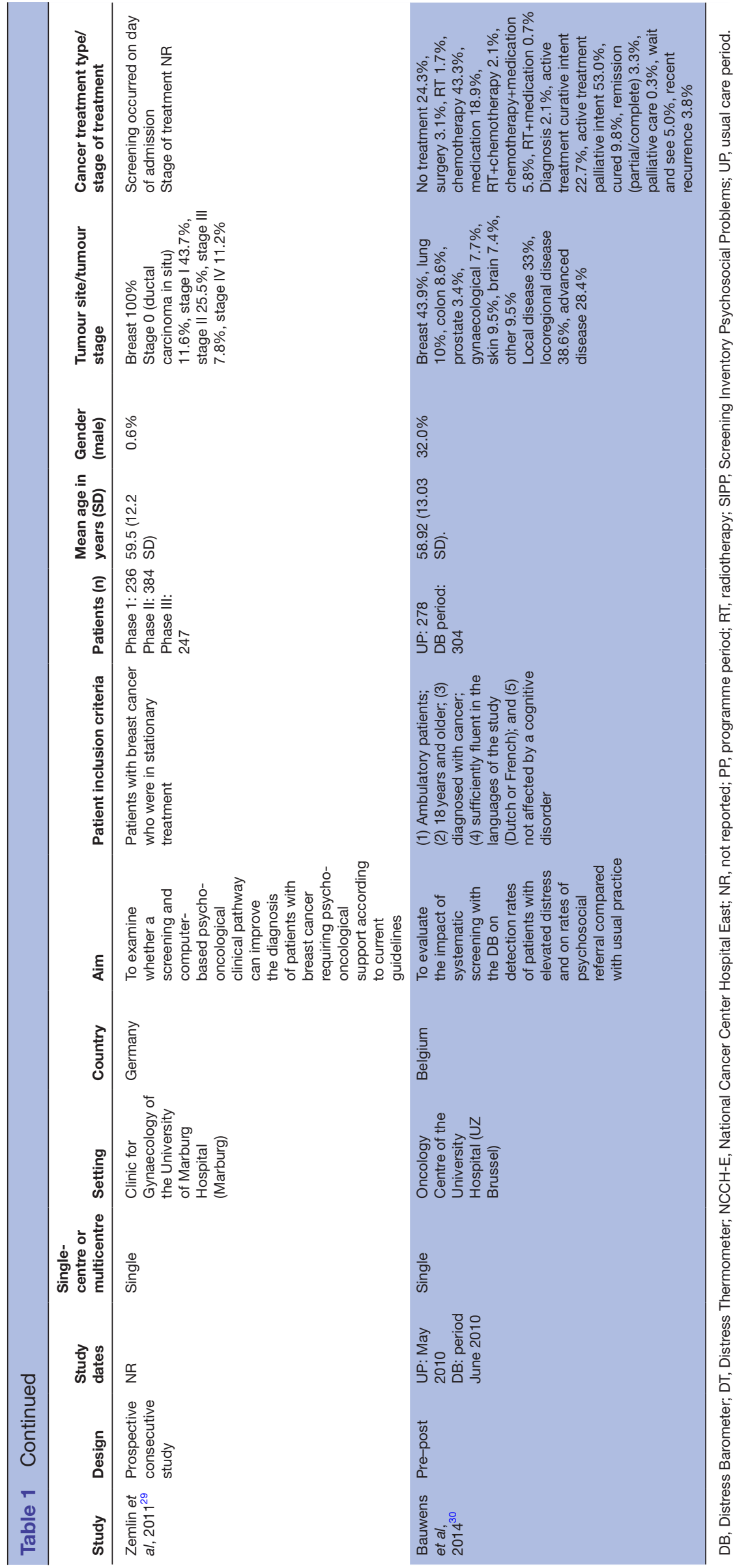




\begin{tabular}{|c|c|}
\hline EPOC subcategory & Definition \\
\hline Educational materials & $\begin{array}{l}\text { Distribution to individuals, or groups, of educational materials to support clinical care, that } \\
\text { is, any intervention in which knowledge is distributed; for example, this may be facilitated } \\
\text { by the internet, learning critical appraisal skills; skills for electronic retrieval of information, } \\
\text { diagnostic formulation; question formulation }\end{array}$ \\
\hline Educational meetings & Courses, workshops, conferences or other educational meetings \\
\hline Reminders & $\begin{array}{l}\text { Manual or computerised interventions that prompt health workers to perform an action } \\
\text { during a consultation with a patient, for example, computer decision support systems }\end{array}$ \\
\hline Organisational culture & Strategies to change organisational culture \\
\hline Continuous quality improvement & $\begin{array}{l}\text { An iterative process to review and improve care that includes involvement of healthcare } \\
\text { teams, analysis of a process or system, a structured process improvement method or } \\
\text { problem-solving approach, and use of data analysis to assess changes }\end{array}$ \\
\hline
\end{tabular}

screening or referral. ${ }^{30} 31$ Thewes $e t a l^{31}$ conducted a pre-post trial testing the feasibility and acceptability of introducing a routine psychological screening programme using the Distress Thermometer (DT) to improve screening rates and timeliness of referral to psychosocial services in three rural outpatient oncology clinics in Australia. Nursing and psychosocial staff participated in a 2-hour training session (educational meetings and educational materials) covering the rationale for screening, the screening instrument and the study procedure. The impact of the intervention on distress screening was not explicitly reported (ie, the control period rates of screening). Five of eight cases (according to predefined PSYCH-6 (psychological symptoms) cut-off criteria) and 10 of 19 cases (according to the DT cut-off) were referred to a social worker or psychologist in the control and intervention periods, respectively. Due to the small number of cases, significance testing of differences between the prescreening and screening phases was not conducted.

Bauwens et $a l^{30}$ conducted a pre-post study to evaluate the impact of systematic screening with the Distress Barometer (DB) on detection rates of elevated distress and on rates of psychosocial referral at an oncology centre in Belgium. Oncologists were instructed in using the DB and given a written explanation (educational materials) on how to interpret the DB results in a collective 1-hour session (educational meetings). As this study did not aim to improve rates of distress screening, but focused on oncologist detection of distress and subsequent referral, all patients were screened using the DB in both conditions. Consequently, the rates of distress screening prior to the study, conducted by oncologists or other professional staff, compared with the study period, are unknown. In the usual care period, using oncologists' judgement, referral was considered necessary for $5.4 \%$ of all patients. In the DB condition, referral was considered necessary for $41.6 \%$ of all patients. Of those patients for whom referral was considered necessary, $40 \%$
$(6 / 15)$ in the usual care period and $69 \%(85 / 123)$ in the DB condition were actually referred to psychosocial care. The authors did not conduct an analysis to determine if there was a significant difference in these rates, however concluded that the implementation of screening using the DB led to increased numbers of referrals to psychosocial professionals.

\section{Educational materials, educational meetings and outreach visits} Braeken et $a l^{26-28}$ conducted a cluster randomised controlled trial to study the effect of the implementation of the Screening Inventory Psychosocial Problems (SIPP) on the number and types of referrals of patients with cancer to psychosocial caregivers in a radiation oncology department in the Netherlands. Radiation oncologists were randomised to a control or intervention group. Those in the intervention group were trained by a researcher and two social workers with experience in using and interpreting the SIPP during a 1-hour training session (educational meetings, educational materials and educational outreach visits). The study found no significant intervention effects were observed for the total number of patients referred to psychosocial care providers at any of the assessment time points (first 3 months, the last 9 months and the total study period).

Educational materials, educational meetings, educational outreach visits and reminders

Ito and colleagues ${ }^{25}$ conducted a pre-post trial to examine the usefulness of a screening programme (using the distress and impact thermometer; DIT) modified for patients with cancer undergoing radiotherapy at an outpatient cancer treatment centre in Japan. Prior to the screening phase, all pharmacists attended a 2-hour lecture and (educational meetings) given by a trained psychiatrist (who also met with the pharmacists monthly; educational outreach visits) and underwent role-play training to learn how to implement the DIT and referral for those patients 


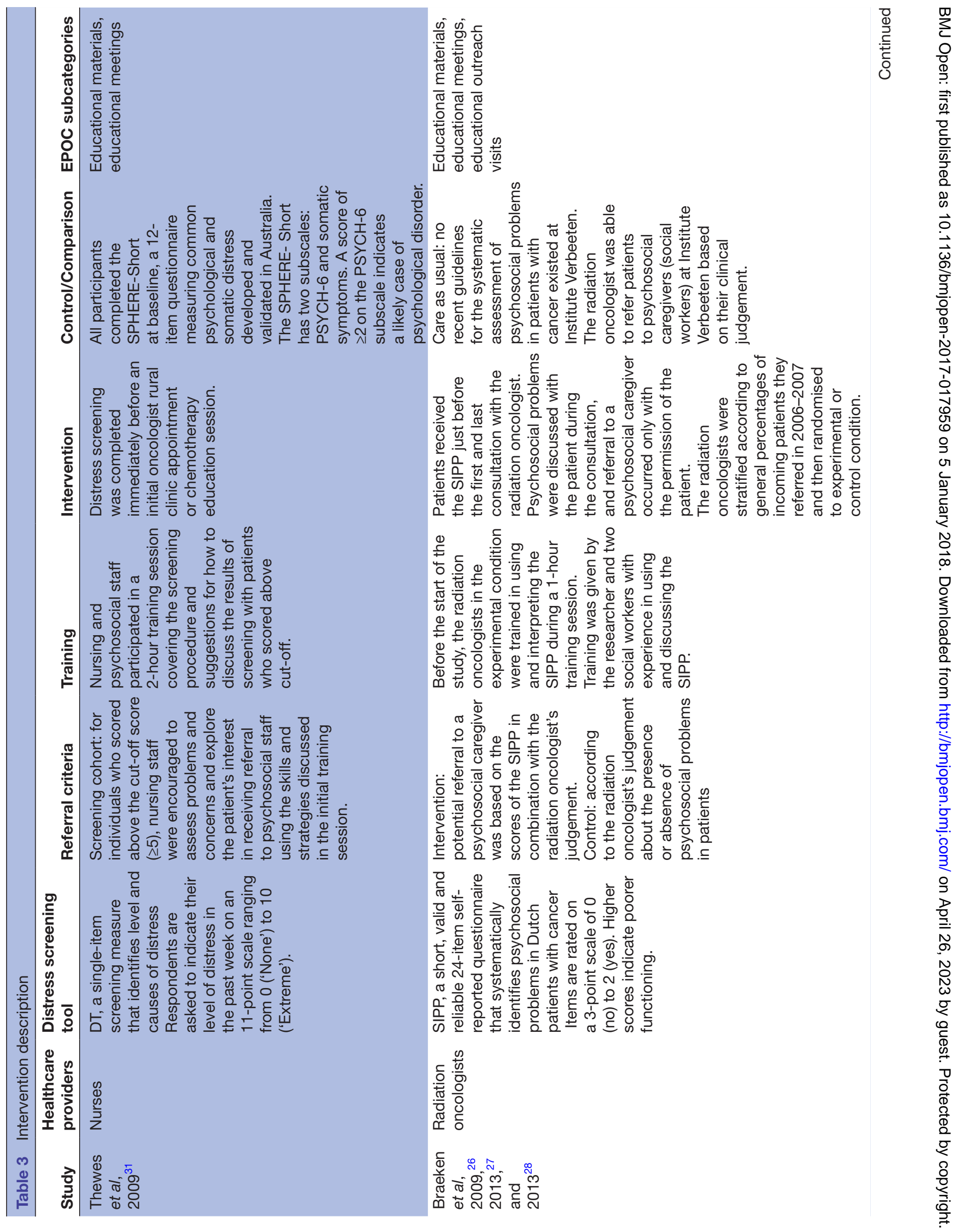




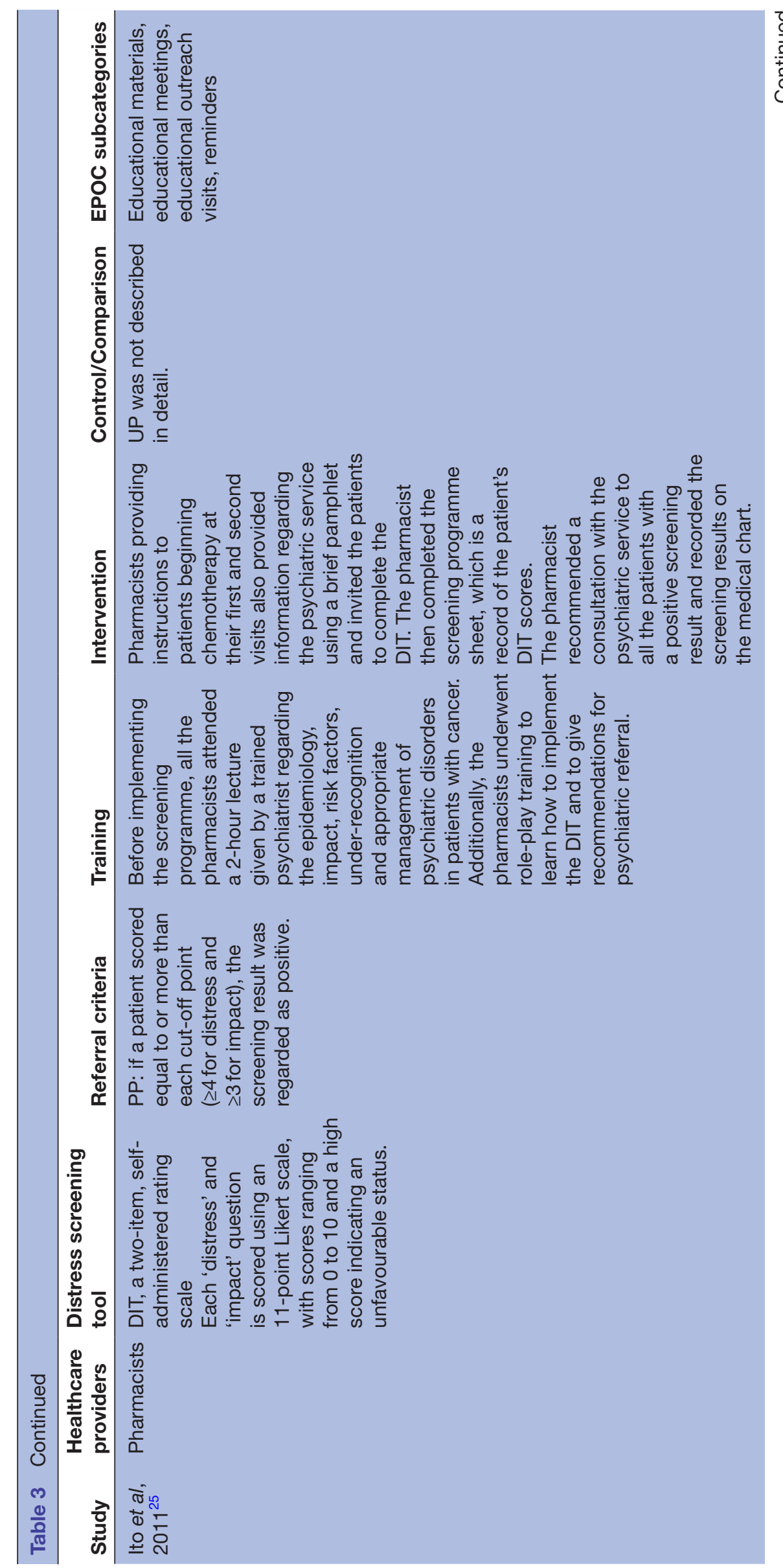




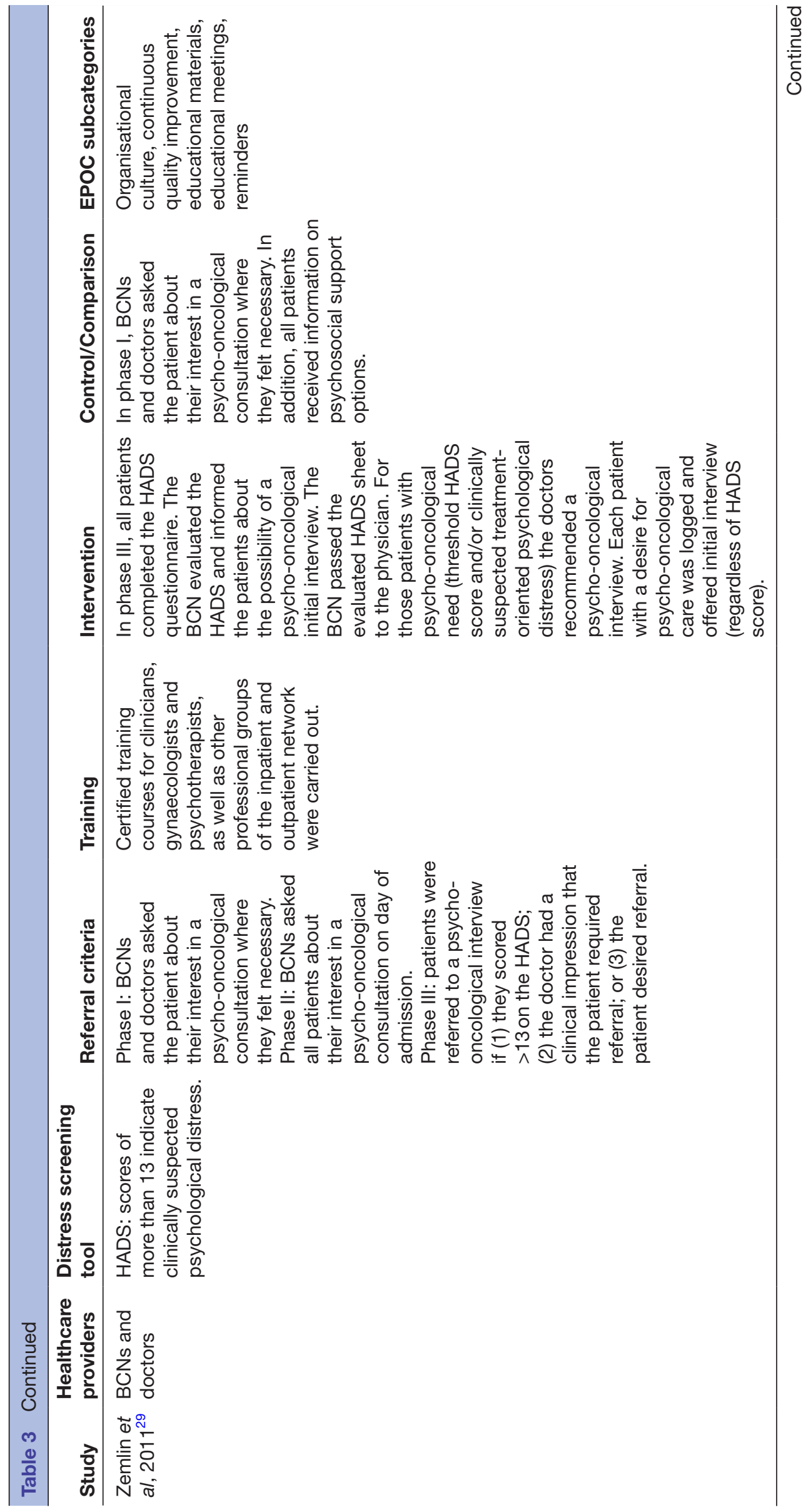



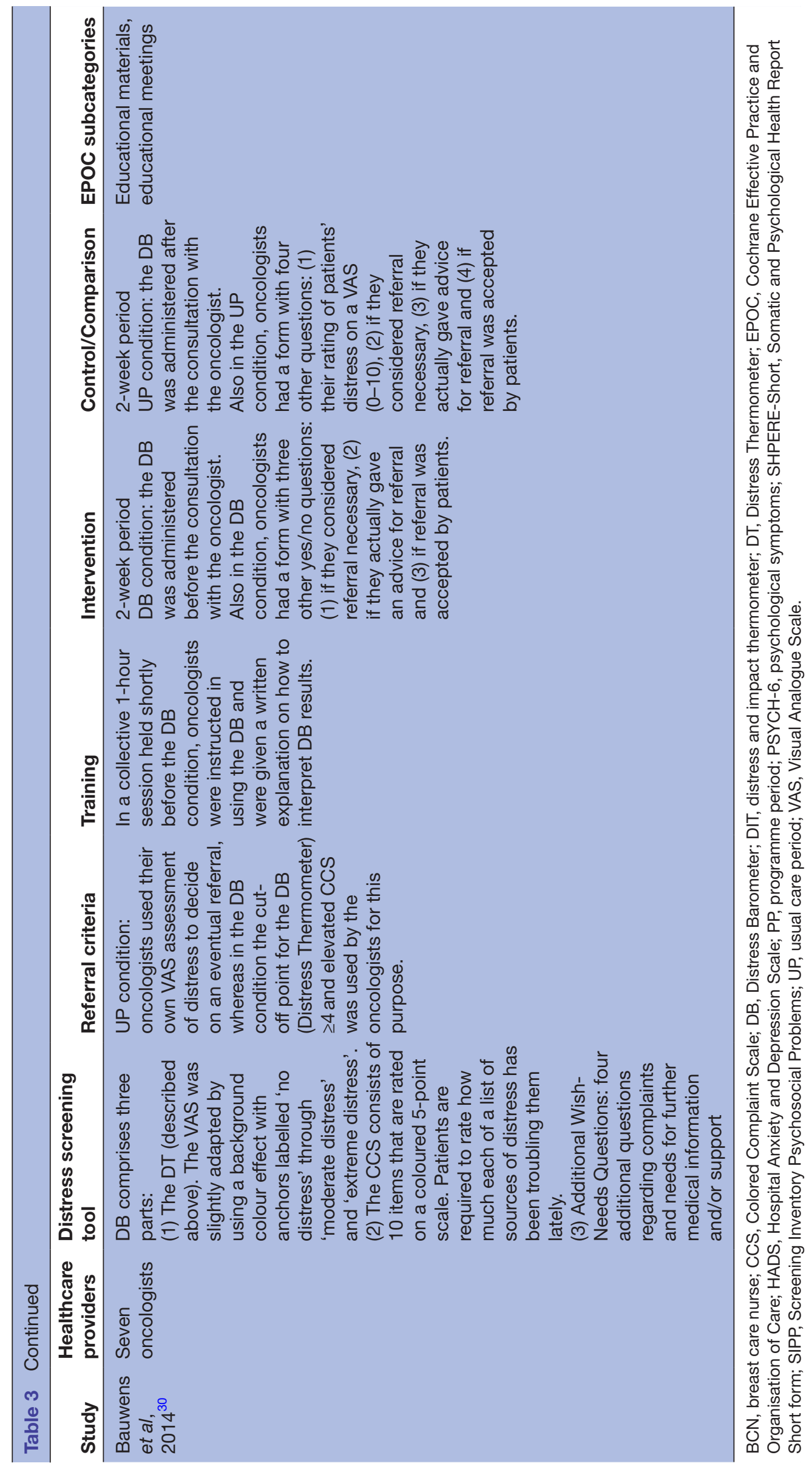
Table 4 Primary outcomes

\begin{tabular}{|c|c|c|c|}
\hline \multirow[b]{2}{*}{ Study } & \multicolumn{2}{|l|}{ Distress screening } & \multirow{2}{*}{$\begin{array}{l}\text { Referral } \\
\text { Measure; data collection } \\
\text { method }\end{array}$} \\
\hline & $\begin{array}{l}\text { Measure; data } \\
\text { collection } \\
\text { method }\end{array}$ & Results & \\
\hline $\begin{array}{l}\text { Thewes et al, } \\
2009^{31}\end{array}$ & $\begin{array}{l}\text { Proportion of } \\
\text { patients screened } \\
\text { NR }\end{array}$ & $\begin{array}{l}\text { Prescreening phase: proportion } \\
\text { of patients screened (using any } \\
\text { distress screening tool) was not } \\
\text { reported. } \\
\text { Screening phase: all patients } \\
\text { were screened using the DT. }\end{array}$ & $\begin{array}{l}\text { Proportion of patients referred } \\
\text { in the prescreening phase } \\
\text { compared with the screening } \\
\text { phase } \\
\text { Review of referral records and } \\
\text { databases }\end{array}$ \\
\hline
\end{tabular}

Results

Prescreening phase: of the 8 PSYCH- 6 cases in the prescreening phase, 6 were referred to a CCC and 5 to a social worker/psychologist. Screening phase: 10/19 (53\%) patients who met the DT cut-off were referred to a social worker or psychologist (11 of 14 PSYCH-6 cases were referred to the $\mathrm{CCC}$ and 8 to a social worker/psychologist).

Braeken et al, Proportion of Control group: proportion of $2009,{ }^{26} 2013^{27}$ patients screened patients screened (using any and $2013^{28} \quad$ NR distress screening tool) was not reported.

Intervention group: 263/268 $(98 \%)$ were screened using the SIPP before the first consultation. 250/268 (96\%) were screened using the SIPP before end of radiotherapy consultation.
The number of referrals of patients with psychosocial problems to psychosocial and/or to external healthcare providers (eg, psychologists, psychiatrists)

Three dichotomous outcome variables (yes/no) during the firs 3 months, the last 9 months and the total study period Measured at 3 and 12 months after baseline assessment with self-developed questionnaire by the patient and from registration records of the psychosocial caregivers at Institute Verbeeten workers at Institute Verbeeten

First 3 months: control group 29/300 $(9.7 \%)$ vs intervention group $34 / 268$ (12.7\%) patients referred (NS)

Last 9 months: control group 24/300

(8\%) vs intervention group 19/268

(7.1\%) patients referred (NS)

Group differences in these outcomes were analysed using generalised estimating equations with patients at level 1 and radiation oncologists at level 2. All models were adjusted for baseline differences with respect to gender and cancer diagnosis.

Analyses were taken on an intentionto-treat principle.

Generalised estimating equations found that numbers of referrals did not differ significantly between the intervention and control group at 3 months $(\beta=-0.16, \mathrm{SE} \pm 0.34, \mathrm{P}=0.32)$, 9 months $(\beta=0.22, S E \pm 0.28, P=0.22)$ or overall months $(\beta=-0.04, \mathrm{SE} \pm 0.28$, $\mathrm{P}=0.44$ ).

$\begin{aligned} \text { Ito et } a l, 2011^{25} & \text { Proportion of } \\ & \text { patients screened } \\ & \text { NR. }\end{aligned}$

UP: proportion of patients screened (using any distress screening tool) was not reported. PP: $441 / 520$ (84.8\%)

Zemlin et al, $2011^{29}$

\section{Proportion of} pat NR.
Proportion of patients screened in phase I or II screened (using any distress screening tool) was not reported.

All patients in phase III were screened using the HADS.
Proportion of patients referred to the psychiatric service and treated for MDD or AD among all the outpatients who had begun a new chemotherapy regimen within 3 months of their visit to the outpatient clinic Data extracted from patients' medical charts and the computerised database of the electronic medical record at $\mathrm{NCCH}-\mathrm{E}$

Retrospective cohort analysis $\chi^{2}$ test comparing patients treated during the PP with historical control data gathered during the UP) UP 5/478 (1.0\%) vs PP $15 / 520$ (2.7\%) patients referred to the psychiatric service subsequently confirmed and treated for MDD or ADs $(\mathrm{P}=0.46)$

Proportion of patients offered referral for psycho-oncological interview Medical records
Univariate data analysis

Cochran-Armitage test

Phase I 194/236 (82.2\%) vs phase II $344 / 384$ (89.6\%) vs phase III 236/247 $(95.5 \%)$ were informed/offered the psycho-oncological interview. There was a significant positive trend for the proportion of patients informed about the psycho-oncological care available $(\mathrm{t}=22.40, \mathrm{df}=2, \mathrm{P}<0.001)$. 
Table 4 Continued

\begin{tabular}{|c|c|c|c|c|}
\hline \multirow[b]{2}{*}{ Study } & \multicolumn{2}{|l|}{ Distress screening } & \multicolumn{2}{|l|}{ Referral } \\
\hline & $\begin{array}{l}\text { Measure; data } \\
\text { collection } \\
\text { method }\end{array}$ & Results & $\begin{array}{l}\text { Measure; data collection } \\
\text { method }\end{array}$ & Results \\
\hline $\begin{array}{l}\text { Bauwens et al, } \\
2014^{30}\end{array}$ & $\begin{array}{l}\text { Proportion of } \\
\text { patients screened } \\
\text { NR }\end{array}$ & $\begin{array}{l}\text { UP condition: all patients } \\
\text { were screened with the DB } \\
\text { after consult with oncologist } \\
\text { (therefore not used as part of } \\
\text { the referral decision). } \\
\text { DB condition: all patients were } \\
\text { screened with the DB prior to } \\
\text { consult with the oncologist. }\end{array}$ & $\begin{array}{l}\text { Necessary referrals (UP } \\
\text { condition: referrals necessary as } \\
\text { per oncologists' VAS ratings; DB } \\
\text { condition: referrals necessary } \\
\text { for all patients with distress } \\
\text { according to the DB) } \\
\text { Self-assessment } \\
\text { Referrals made (UP condition: } \\
\text { proportion of patients for } \\
\text { whom referral was considered } \\
\text { necessary by the oncologists } \\
\text { and were actually referred to } \\
\text { psychosocial care; DB condition: } \\
\text { proportion of patients with } \\
\text { elevated distress who were } \\
\text { referred) } \\
\text { Self-assessment }\end{array}$ & $\begin{array}{l}\text { UP condition: } 13.8 \% \text { of patients } \\
\text { with elevated distress (or } 5.4 \% \text { of } \\
\text { all patients); DB condition: } 100 \% \text { of } \\
\text { patients with distress (or } 41.6 \% \text { of all } \\
\text { patients) } \\
\text { UP condition: } 6 / 15 \text { patients; DB } \\
\text { condition: } 85 / 123 \text { patients }\end{array}$ \\
\hline
\end{tabular}

AD, adjustment disorder; CCC, cancer care coordinator; DB, Distress Barometer; DT, Distress Thermometer; HADS, Hospital Anxiety and Depression Scale; MDD, major depressive disorder; NCCH-E, National Cancer Center Hospital East; NR, not reported; NS, not significant.; PP, programme period; PSYCH-6, psychological symptoms; SIPP, Screening Inventory Psychosocial Problems; UP, usual care period; VAS, visual analogue scale.

scoring above the predetermined cut-off (educational materials). When providing instructions to patients beginning chemotherapy and at the second visit, pharmacists invited patients to complete the DIT and a screening programme sheet was completed by the pharmacists (reminders). The proportion of patients screened prior to the implementation of the screening programme using the DIT or other measure was not assessed, and $84.8 \%$ of patients were screened using the DIT in the intervention phase. The proportion of patients referred to the psychiatric service (and were subsequently confirmed to have major depression or adjustment disorder) during the screening programme period compared with the usual care period was not significantly different between the two periods $(2.7 \%$ during the programme period vs $1.0 \%$ during the usual care period; $\mathrm{P}=0.46$ ).

Educational materials, educational meetings, reminders, organisational culture and continuous quality improvement One study examined the effect of educational materials, educational meetings, reminders, organisational culture and continuous quality improvement on improvement in distress screening or referral. The trial by Zemlin $e t a t^{29}$ was a prospective consecutive study that aimed to integrate psycho-oncological early detection and diagnostics

\section{Table 5 Secondary outcomes}

\begin{tabular}{|c|c|c|}
\hline Study & Measure; data collection method & Results \\
\hline \multirow{11}{*}{$\begin{array}{l}\text { Braeken et al, } \\
2009,{ }^{26} 2013^{27} \\
\text { and } 2013^{28}\end{array}$} & Extent of psychological symptoms at 3 months and & Mixed effects modelling \\
\hline & 12 months after baseline & No significant intervention effects were observed \\
\hline & Measured with the HADS and the GHQ-12 (assesses & \\
\hline & or herself better, the same, worse or much worse & distress score control group 2.85 vs intervention \\
\hline & $\begin{array}{l}\text { over the previous } 4 \text { weeks than he/she 'usually' is; } \\
\text { total scores range from } 0 \text { to } 12 \text { ) }\end{array}$ & $\begin{array}{l}\text { group } 2.74, \mathrm{P}=0.19 ; 12 \text { months after baseline mean } \\
\text { psychological distress score control group } 2.14 \text { vs }\end{array}$ \\
\hline & $\begin{array}{l}\text { Patients complete these self-reported questionnaires } \\
\text { at baseline and at } 3 \text { and } 12 \text { months after the baseline } \\
\text { period. }\end{array}$ & intervention group 1.96, $\mathrm{P}=0.12)$ \\
\hline & \multirow{5}{*}{$\begin{array}{l}\text { Group differences in the proportion of dichotomous } \\
\text { distress outcome (no or at least moderate distress) } \\
\text { at } 3 \text { months and } 12 \text { months after baseline } \\
\text { Measured with HADS and GHQ-12 }\end{array}$} & Generalised estimating equations \\
\hline & & No significant intervention effects were observed for \\
\hline & & proportion of patients with distress ( 3 months after \\
\hline & & baseline control group 39\% vs experimental group \\
\hline & & $\begin{array}{l}38.4 \%, P=0.036 ; 12 \text { months after baseline control } \\
\text { group } 24.7 \% \text { vs intervention group } 24.3 \%, P=0.39 \text { ). }\end{array}$ \\
\hline
\end{tabular}

GHQ-12, Goldberg's General Health Questionnaire-12 item version; HADS, Hospital Anxiety and Depression Scale. 
Table 6 Ratings of methodological quality: strong, moderate and weak

\begin{tabular}{|c|c|c|c|c|c|c|c|}
\hline Study & $\begin{array}{l}\text { Selection } \\
\text { bias }\end{array}$ & Study design & Confounders & Blinding & $\begin{array}{l}\text { Data } \\
\text { collection }\end{array}$ & Withdrawals & $\begin{array}{l}\text { Global } \\
\text { rating }\end{array}$ \\
\hline Thewes et al, $2009^{31}$ & Moderate & Moderate & Weak & Moderate & Weak & Moderate & Weak \\
\hline Ito et al, $2011^{25}$ & Moderate & Moderate & Strong & Moderate & Weak & Moderate & Moderate \\
\hline Zemlin et al, $2011^{29}$ & Moderate & Moderate & Weak & Moderate & Weak & Moderate & Weak \\
\hline
\end{tabular}

as an integral part of everyday practice routines of acute inpatient care within the multidisciplinary diagnosis and care chain of patients with breast cancer at a gynaecology clinic in Germany. Prior to the introduction of the programme, certified training courses were held for clinicians, gynaecologists and psychotherapists, as well as other professional groups (educational meetings, educational materials, organisational culture), and every 3-4 months cross-departmental meetings between psychology and gynaecology departments were held (continuous quality improvement). The authors described the trial in three phases: in phase I, breast care nurses and doctors asked the patient about their interest in a psycho-oncological consultation where they felt necessary, and in phase II the nurses asked this of patients on the day of their admission. In phase III, the nurses conducted screening using the Hospital Anxiety and Depression Scale (HADS) with all patients and passed the HADS sheet to the physician (reminders). A predetermined cut-off indicated if referral was required. The proportion of patients screened with the HADS during phase III was $100 \%$. The proportion of patients screened in phase I or II using the HADS or other measure was not assessed. The authors reported a significant positive trend for the proportion of patients offered referral for psycho-oncological care between phases I and III $(\mathrm{t}=22.40, \mathrm{df}=2, \mathrm{P}<0.001)$.

\section{Secondary outcomes}

Psychosocial distress

Only one study compared patients' levels of distress at follow-up using the distress screening measure implemented. Braeken et $a l^{26-28}$ found no significant intervention effects as measured by the HADS for patients' psychological distress at 3 months or 12 months after baseline, nor dichotomous distress outcomes (no distress or at least moderate distress) at 3 months or 12 months after baseline.

\section{Reported adverse consequences}

No study explicitly assessed whether the intervention had adverse effects.

\section{Quality of the evidence}

Using GRADE, the overall rating of the certainty of the body of evidence reported in this review was assessed as very low. The primary outcomes examined were downgraded one level to reflect high risk of bias and further downgraded two levels due to clinical heterogeneity and inconsistency in reporting either rates of distress screening or referral across both control and intervention periods. Since indirectness and imprecision also lower the quality of the evidence, we downgraded two further levels on that basis. We found the quality of evidence to be of weak to moderate quality due to risk of bias using the EPHPP (table 6), which identified a number of limitations, particularly among the pre-post studies in regard to controlling for potential confounders.

\section{DISCUSSION}

This review sought to assess the impact of trials of strategies to improve clinician provision of screening of patients with cancer for psychosocial distress, and referral for further assessment and/or psychosocial support where necessary. The review identified just one trial that met the prospectively registered inclusion criteria of having a parallel control trial design. When these criteria were relaxed to include those with a non-parallel control group, a further four trials were included. Largely due to study designs (ie, mostly pre-post), none of the included studies were able to provide quality evidence for the effectiveness of screening procedures in improving rates of distress screening. The intervention in just one trial was effective in significantly improving the rates of referral for psycho-oncological support for distressed patients. Such findings highlight the sparse evidence base for this important element of care for patients with cancer, and leave health services and cancer professionals with little clear guidance of strategies to improve provision of these elements of care to their patients.

Our findings are consistent with previous systematic reviews of trials aiming to improve depression or anxiety screening in primary care that have found that improvement in care provision is more likely when complex organisational change strategies are used, such as coordination between departments, enhanced role of nurses and performance feedback, in addition to clinician education. ${ }^{13-15}$ The findings of the review highlight that the implementation of routine psychosocial screening and referral in cancer is complex and more rigorous research is needed. The trial by Zemlin et $a l^{29}$ was the only study 
included in the review to adopt a comprehensive implementation approach, and the only study to report significant improvement in offer of referral of patients with cancer for distress. Implementation strategies employed by other trials were primarily based on one-off training and resource provision, suggesting that such support is insufficient. Comprehensive implementation strategies may be more likely to improve care given their greater capacity to address various barriers to screening and referral. Interestingly, Zemlin $e t a l^{29}$ was the only study to describe strategies employed to change the organisational culture of the healthcare setting, specifically defining responsibilities and tasks between the specialist disciplines and the medical and nursing staff involved in the treatment team, training certificates, as well as regular meetings to facilitate communication. It may be that simpler interventions are less effective in implementing routine provision of this care because they fail to address the organisational culture of the setting. Strengthening team communication ${ }^{25}$ and making clinicians more aware of their role and responsibilities in distress screening and referral for patients with cancer ${ }^{27}$ may improve the rates of this care delivery. Further research identifying the key barriers to such care and the best strategies to address them in cancer services is therefore warranted.

Surprisingly, none of the included studies examined the impact of strategies employed (eg, training) to improve the rate of clinician provision of psychosocial distress screening. Due to the majority of study designs not employing a parallel comparison group, the review does not provide quality evidence regarding the effectiveness of implementation strategies to improve screening or referral. Such a finding is of concern. Screening is a necessary prerequisite to appropriate referral of patients with cancer to psychological support. As screening for psychosocial distress in cancer populations is low across jurisdictions, ${ }^{32}$ improving this form of care should represent a priority. Previous studies have used novel technologies to prompt screening by clinicians. ${ }^{33-35}$ Such approaches should be examined in robust trial designs in cancer settings that allow for their impact on improving the rate of routine clinician provision of distress screening to be determined.

A number of methodological aspects of the study warrant highlighting and should be considered when interpreting the study findings. As far as the authors are aware, this is the first systematic review to examine the impact of interventions of strategies to improve the rate of clinician provision of distress screening and appropriate referral in patients with cancer. The review was prospectively registered, followed a peer-reviewed protocol and included a comprehensive search strategy examining over 18000 citations. There was substantial clinical and methodological heterogeneity in the included studies. Classification of EPOC taxonomy implementation strategies was also difficult due to the lack of detail reported on intervention components in the studies. Furthermore, only one of the studies was a randomised controlled trial.
Such characteristics of the included studies precluded quantitative synthesis of the effects of these strategies.

\section{CONCLUSIONS}

The findings of this review suggest that there is considerable scope to improve implementation of psychosocial distress screening and referral in cancer settings in order to establish a strong evidence base for future successful interventions. Implementation of psychosocial distress screening and appropriate referral needs to be employed using a systematic method and assessed with appropriately controlled studies in order to determine the most effective approaches. Better reporting of outcomes and more detailed description of intervention components need to be prepared.

Contributors KM and LW conceptualised the review with input from BB, ALB, SAH, $\mathrm{AKB}, \mathrm{GC}, \mathrm{CW}, \mathrm{JB}, \mathrm{DB}$ and EF. KM and EF conducted screening, data extraction and methodological quality analysis. KM and LW drafted the manuscript. All authors contributed to subsequent drafts and have approved the final version of the manuscript.

Funding This work was supported by a Hunter Cancer Research Alliance Implementation Flagship Program grant.

Competing interests None declared.

Provenance and peer review Not commissioned; externally peer reviewed.

Data sharing statement № additional data available.

Open Access This is an Open Access article distributed in accordance with the Creative Commons Attribution Non Commercial (CC BY-NC 4.0) license, which permits others to distribute, remix, adapt, build upon this work non-commercially, and license their derivative works on different terms, provided the original work is properly cited and the use is non-commercial. See: http://creativecommons.org/ licenses/by-nc/4.0/

(c) Article author(s) (or their employer(s) unless otherwise stated in the text of the article) 2018. All rights reserved. No commercial use is permitted unless otherwise expressly granted.

\section{REFERENCES}

1. National Comprehensive Cancer Network. NCCN clinical practice guidelines in oncology: distress management, 2017.

2. Ma L, Poulin P, Feldstain A, et al. The association between malnutrition and psychological distress in patients with advanced head-and-neck cancer. Curr Oncol 2013;20:554-60.

3. Holland JC, Alici Y. Management of distress in cancer patients. $J$ Support Oncol 2010;8:4-12.

4. Bultz BD, Carlson LE. Emotional distress: the sixth vital sign--future directions in cancer care. Psychooncology 2006;15:93-5.

5. Jacobsen P, Donovan K, Swaine Z. et alManagement of anxiety and depression in adult cancer patients: toward an evidence-based approach. In: Chang A, Ganz P, Hayes D, Kinsella T, Pass H, . eds. Oncology: an evidence-based approach. New York: Springer-Verlag, 2006:1552-79.

6. Barsevick AM, Sweeney C, Haney E, et al. A systematic qualitative analysis of psychoeducational interventions for depression in patients with cancer. Oncol Nurs Forum 2002;29:73-87. quiz 5-7.

7. Osborn RL, Demoncada AC, Feuerstein M. Psychosocial interventions for depression, anxiety, and quality of life in cancer survivors: meta-analyses. Int J Psychiatry Med 2006;36:13-34.

8. Andrykowski MA, Manne SL. Are psychological interventions effective and accepted by cancer patients? I. Standards and levels of evidence. Ann Behav Med 2006;32:93-7.

9. Meijer A, Roseman M, Delisle VC, et al. Effects of screening for psychological distress on patient outcomes in cancer: a systematic review. J Psychosom Res 2013;75:1-17.

10. Mitchell AJ. Screening for cancer-related distress: when is implementation successful and when is it unsuccessful? Acta Oncol 2013;52:216-24. 
11. Jacobsen PB, Ransom S. Implementation of NCCN distress management guidelines by member institutions. J Natl Compr Canc Netw 2007;5:99-103.

12. American College of Surgeons Commission on Cancer. Cancer program standards 2012 version 1.2.1: ensuring patient-centered care, 2012.

13. Hermanns N, Caputo S, Dzida G, et al. Screening, evaluation and management of depression in people with diabetes in primary care. Prim Care Diabetes 2013;7:1-10.

14. Gilbody S, Whitty P, Grimshaw J, et al. Educational and organizational interventions to improve the management of depression in primary care: a systematic review. JAMA 2003;289:3145-51.

15. Heideman J, van Rijswijk E, van Lin N, et al. Interventions to improve management of anxiety disorders in general practice: a systematic review. Br J Gen Pract 2005;55:867-73.

16. Moher D, Liberati A, Tetzlaff J, et al. Preferred reporting items for systematic reviews and meta-analyses: the PRISMA statement. $J$ Clin Epidemiol 2009;62:1006-12.

17. McCarter K, Britton B, Baker A, et al. Interventions to improve screening and appropriate referral of patients with cancer for distress: systematic review protocol. BMJ Open 2015;5:e008277.

18. Effective Practice and Organisation of Care (EPOC). EPOC taxonomy, 2015.

19. Ostuzzi G, Matcham F, Dauchy S, et al. Antidepressants for the treatment of depression in people with cancer. Cochrane Database Syst Rev 2015:CD011006.

20. Vodermaier A, Linden W, Siu C. Screening for emotional distress in cancer patients: a systematic review of assessment instruments. J Natl Cancer Inst 2009;101:1464-88.

21. Akbari A, Mayhew A, Al-Alawi MA, et al. Interventions to improve outpatient referrals from primary care to secondary care. Cochrane Database Syst Rev 2008;4:CD005471.

22. Bower P, Knowles S, Coventry PA, et al. Counselling for mental health and psychosocial problems in primary care. Cochrane Database Syst Rev 2011:CD001025.

23. Higgins JP, Green S. Cochrane handbook for systematic reviews of interventions. Wiley Online Library, 2008.

24. Armijo-Olivo S, Stiles CR, Hagen NA, et al. Assessment of study quality for systematic reviews: a comparison of the cochrane collaboration risk of bias tool and the effective public health practice project quality assessment tool: methodological research. J Eval Clin Pract 2012;18:12-18.

25. Ito T, Shimizu K, Ichida $\mathrm{Y}$, et al. Usefulness of pharmacist-assisted screening and psychiatric referral program for outpatients with cancer undergoing chemotherapy. Psychooncology 2011;20:647-54.

26. Braeken AP, Lechner L, van Gils FC, et al. The effectiveness of the Screening Inventory of Psychosocial Problems (SIPP) in cancer patients treated with radiotherapy: design of a cluster randomised controlled trial. BMC Cancer 2009;9:177.

27. Braeken AP, Lechner L, Eekers DB, et al. Does routine psychosocial screening improve referral to psychosocial care providers and patient-radiotherapist communication? A cluster randomized controlled trial. Patient Educ Couns 2013;93:289-97.

28. Braeken AP, Kempen GI, Eekers DB, et al. Psychosocial screening effects on health-related outcomes in patients receiving radiotherapy. A cluster randomised controlled trial. Psychooncology 2013;22:2736-46.

29. Zemlin C, Herrmann-Lingen C, Wiegard K, et al. Implementation of a computer and screening-based psycho-oncological clinical pathway. Geburtshilfe und Frauenheilkunde 2011;71:853-61.

30. Bauwens S, Baillon C, Distelmans W, et al. Systematic screening for distress in oncology practice using the Distress Barometer: the impact on referrals to psychosocial care. Psychooncology 2014;23:804-11.

31. Thewes B, Butow P, Stuart-Harris R. Greater Southern Area Health Service Screening Collaborative Group. Does routine psychological screening of newly diagnosed rural cancer patients lead to better patient outcomes? Results of a pilot study. Aust J Rural Health 2009;17:298-304.

32. Lazenby M, Ercolano E, Grant M, et al. Supporting commission on cancer-mandated psychosocial distress screening with implementation strategies. J Oncol Pract 2015;11:e413-20.

33. Wolfenden L, Wiggers J, Knight J, et al. Increasing smoking cessation care in a preoperative clinic: a randomized controlled trial. Prev Med 2005;41:284-90.

34. Wolfenden L, Wiggers J, Campbell E, et al. Feasibility, acceptability, and cost of referring surgical patients for postdischarge cessation support from a quitline. Nicotine Tob Res 2008;10:1105-8.

35. Dexheimer JW, Talbot TR, Sanders DL, et al. Prompting clinicians about preventive care measures: a systematic review of randomized controlled trials. J Am Med Inform Assoc 2008;15:311-20. 